\title{
Bilateral and Multilateral Mechanisms in Comparison: An Examination Through China's Climate Policy
}

\author{
Mohan Chang, ${ }^{1, *}$ \\ ${ }^{1}$ School of Foreign Languages, Jimei University, Xiamen, 361001, China \\ ${ }^{*}$ Corresponding author. Email: 201921152064@jmu.edu.cn
}

\begin{abstract}
The environment has been an important issue for a long time. All the countries have different policies to treat these problems. In general, all international policies operate under certain mechanism, especially bilateral and multilateral mechanisms. Then what are the pros and cons of these two mechanisms for countries in international relations? The article will try to answer this question by providing an in-depth comparative analysis of China's bilateral and multilateral mechanisms related to environmental policies. Both bilateral mechanisms and multilateral mechanisms have advantages and disadvantages. Through the case of China this analysis shows that it would be beneficial for countries to use a combination of both, leveraging the advantages of both mechanisms simultaneously.
\end{abstract}

Keywords: Bilateral mechanisms, Multilateral mechanisms, Climate Change, Environmental Policy, Cooperation, China, the United States, the European Union, the United Nations

\section{INTRODUCTION}

Bilateral and multilateral mechanisms are very important channels in international relations. A bilateral mechanism is one in which two countries cooperate directly for mutual benefit. In 1990, Dr. Robert O. Keohane defined multilateralism as the practice of coordinating national policies among three or more countries or groups [1]. And in 1992, John Gerard Rudge built on the definition of multilateralism by suggesting that multilateralism requires a rules-based international order, thus laying the groundwork for the creation of a multilateral mechanism [2]. Multilateralism emphasizes the involvement of third parties, allowing groups to limit the participants and vested interests involved in the game. And it makes the outcome of decisions subject to the influence of each party. Multilateral mechanisms are also considered to be an expression of collectivism.

In the previous studies on environmental policy, bilateral mechanisms have been considered important means of cooperation, such as the U.S.-China summit on Carbon Capture, the U.S.-China Joint Declaration on Climate between China and the United States (US), and the biodiversity workshops between China and the European Union (EU). However, in recent years, multilateral mechanisms have been increasingly used as globalization takes hold. The Paris Agreement is a good example of the application of multilateral mechanisms in climate change policy.

How do the multilateral mechanisms is operationalized with regards to environmental policies, especially in comparison to the bilateral mechanism? This research will address the issue by examining China's environmental policies, including China's bilateral environmental protection cooperation policies with other countries, and the newly proposed Green Belt and Road Initiative. Especially, the research will address the questions that how these two mechanisms are implemented in China and how they impact China's international relations, specifically with EU and US.

The article is structured in the following way. Firstly, this paper will provide a background on the use of bilateral and multilateral mechanisms in the environmental policies. Secondly, it will compare bilateral and multilateral mechanisms through the case of Chinese environmental policies. Lastly, the article will be concluded with a discussion on the limitation of this study and future prospects for countries to use bilateral and/or multilateral mechanisms. 


\section{BILATERAL AND MUITILATERAL MECHANISMS}

\subsection{Background}

Bilateral and multilateral mechanisms are effective means for many countries to cooperate with other countries and solve international problems, especially on the environmental issues. Globalization is the most important basic condition for the formation of bilateral and multilateral mechanisms. With the gradual deepening of globalization, countries have deepened their contacts in the political, economic, and cultural fields and have gradually become inseparable. It is precisely because of the need for such cooperation that bilateral and multilateral mechanisms have emerged [3]. At the same time, according to different cooperation projects and purposes, international relations change, and countries will make trade-offs and choices between bilateral and multilateral mechanisms. In recent years, unilateral mechanisms have fallen out of fashion. At one period the EU extended its carbon emissions trading scheme to aviation, levying fines on any aircraft taking off or landing at airports in EU countries that exceeded the Kyoto Protocol standards. To some extent, this did reduce emissions, but it attracted the displeasure of many countries [4]. In the face of all the discontent, the EU has also launched a dialogue with other countries. It can be said that with the development of the economy, countries do not want to follow the previous unilateral mechanisms and they prefer dialogue and exchange. The shift from unilateral to bilateral and multilateral mechanisms is therefore inevitable.

In the past few years, many scholars have written on the bilateral and multilateral mechanisms in international relations, but much of the scholarly attention has been on finance, investment, and trade, while environmental policy has been understudied. Mr. Zhang Rui, Mr. Zhou Yuanbing, and Mr. Xiang Junyong who are from Global Internet Economy Development Cooperation Organization have written an article called International Energy Bilateral and Multilateral Mechanisms Revisited, analyzing bilateral and multilateral mechanisms in the energy field. They classify and analyze the pros and cons of bilateral and multilateral mechanisms in the international energy regime and provide solid recommendations for future changes in China's energy strategy [5]. In the study of mechanisms in the field of environmental policy, Dr. Gao Xiang, Dr. Wang Wentao, and Mr. Dai Yande did a study on the impact of nonconvention mechanisms on the United Nations Framework Convention on Climate Change (UNFCCC). In the article by Dr. Gao Xiang, Dr. Wang Wentao, and Mr. Dai Yandei, they analyzed the characteristics and interrelationships of these mechanisms and the impacts of these non-conventional multilateral mechanisms on the UNFCCC. The importance of the UNFCCC for environmental policy is emphasized [6]. In his master's thesis, Cooperation and Conflict: China and the United States in Global Environmental Governance, Mr. Hu Wei analyzed the process and operational mechanism of global climate governance, focusing on the cooperation and conflict between China and the United States, and worked to identify the key to solving environmental governance problems [7].

China has used both bilateral and multilateral mechanisms with other countries for environmental issues. For example, China and America have had many bilateral mechanisms on climate change since the 1980s. Numerous bilateral agreements have been signed, including U.S.-China Joint Statement on Climate Change signed in 2014, a commitment made by the two countries to work together to reduce greenhouse gas emissions [8]. This bilateral agreement is of great significance. This joint statement is a collaborative effort between two of the world's major emitters to reduce carbon emissions, and its signing will help accelerate the achievement of Carbon peak and Carbon-neutral plans. And for multilateral mechanisms, the United Nations (UN) offers many opportunities for cooperation in multilateral mechanisms for environmental policy. Sometimes there will be some unexpected results, but most of the time these results are positive. China has been actively participated in many UN multilateral mechanisms, such as UN Climate Change Conference Paris 2015, Global Biodiversity Conference.

China still has many environmental problems, air pollution being the first. For China itself, the government adjusted the energy structure and implement Three-Year Action Plan for Blue Sky Defense. What is more, China has actively responded to the call of the UN Climate Change Conference. The Chinese government has also developed policies to achieve carbon peaking and carbon neutrality. In the past years, China mainly uses bilateral mechanisms for environmental policy. But in recent years, China has a trend towards multilateral mechanisms, Because China has gained a deeper and deeper understanding of cooperation realized through multilateral mechanisms. China has put forward the values of a Community of Shared Future for Mankind since 2012. Climate change is not a problem caused by only one or two countries. All the countries have the responsibility to protect the climate. Only cooperation can change the future.

\subsection{Comparative Analysis of Bilateral and Multilateral Mechanisms}

In the past, external cooperation took place through only one of the bilateral or multilateral mechanisms, and perhaps. But nowadays people are increasingly using both at the same time, and they are now seen as a complementary relationship. How do countries decide which mechanism to use? Which mechanisms will be 
used in which situations? Can they be applied simultaneously? It will be the most important to be discussed in follow.

\subsubsection{Bilateral Mechanisms for China's Environmental Policies}

Since the relationships between EU and China and between US and China are very important for international relations, China's bilateral ties with EU and US will be used as examples to illustrate the advantages and disadvantages of bilateral mechanism. Much of China's cooperation with the EU in environmental bilateral mechanisms is a spin-off from the international climate convention. For example, four China-EU biodiversity workshops have been carried out between China and the EU up to 2021, which are derivatives based on the Convention on Biological Diversity. This workshop provides an impetus for China and the EU to work on biodiversity commitments, the 2030 targets, and to better support the Convention on Biological Diversity.

The earliest bilateral mechanisms cooperation between China and the United States is from the last century. The first formal document in the field of environmental diplomacy between China and the United States, the China-United States Protocol on Environmental Protection Cooperation, was signed in the 1980s, and since then China and the United States have made great progress in the bilateral mechanism for environmental protection, for example, the U.S.-China Cooperation on Carbon Capture, U.S.-China Joint Statement on Climate Change. Furthermore, many bilateral intents for cooperation or bilateral seminars may develop into formal bilateral cooperation in the future, such as the US-China Seminar on Environment and Development, the Letter of Intent for Cooperation on the U.S.-China Urban Air Quality Monitoring Project, the U.S.-China Seminar on Environment and Development.

There are advantages and disadvantages to cooperation in bilateral mechanisms.

\subsubsection{1 advantages}

Easier to communicate and coordinate between two countries, bilateral mechanism can be more intuitive and effective for problem solving. The four biodiversity workshops between China and the EU are complementary to the UN Convention on Biological Diversity. Direct dialogue between China and EU are more conducive to cooperation, and at the 2018 ChinaEU Summit, the two sides strengthened bilateral cooperation in multiple areas, including clean energy, low-emissions transport, low-carbon cities, climaterelated technologies, and investment in climate clean energy projects, to enhance the implementation of the Paris Agreement [9].
Implementation through bilateral mechanisms is more binding for both parties than through multilateral mechanisms. Cooperation under bilateral mechanisms is usually guaranteed by agreement. If one party defaults, it will certainly be held accountable. The U.S.-China Joint Statement on Climate Change proposed in 2014 explicitly mentions that both sides will work together to reach an agreed outcome with legally binding effect at UN Climate Change Conference Paris 2015[10].

\subsubsection{2 disadvantages}

Bilateral mechanisms are sometimes susceptible to the changes in international relations. The trade war between the US and China has had an impact on the cooperation between the two on environmental policies. Currently, China is still dependent on the main source of energy, mainly coal. As a result of this, China has experienced unprecedented air pollution in the last few years. Therefore, the transition and development of carbon use are important to the environmental issues in China. Carbon Capture and Storage (CCS) is considered by a wide range of scholars and scientists as an effective way to reduce greenhouse gas emissions and mitigate global warming. CCS is a technology that captures carbon dioxide (CO2) from large power plants and stores it in various ways to prevent it from being released into the atmosphere. In 2015, China and the United States have cooperated on a bilateral mechanism for Carbon Capture projects. However, due to the Sino-US trade war, the relationship between China and the US has cooled down, leading to the suspension of cooperation in many areas.

Bilateral mechanisms can lead to both parties forgetting their true intentions when it comes to environmental cooperation. There are times when the center of bilateral cooperation between the two countries is no longer the environmental improvement and environmental benefits. Such a shift would significantly reduce the effectiveness of cooperation in bilateral mechanisms. The EU has been committed to and has contributed significantly to the improvement of the world's environment. It has actively promoted the landing and formation of the United Nations Framework Convention on Climate Change and the Kyoto Protocol. At the same time, it has actively cooperated with China to establish the China-EU Climate Change Partnership and expand its influence [11]. But while the EU seeks to improve the environment, it also wants to enhance its international status and gain a greater voice through developments in the field of climate change.

\subsubsection{Multilateral mechanisms for China's Environmental Policies}

There are many different classifications of multilateral mechanisms in different contexts, such as 
multilateral benefit-sharing mechanisms, multilateral coordination mechanisms, multilateral consultation mechanisms, and multilateral trade mechanisms. The multilateral mechanisms referred to in this analysis are multilateral in the broadest sense.

Multilateral mechanisms introduced third parties into the bilateral parties so that the relationship between each two parties is more balanced. Multilateral mechanisms can help ease tensions among the three parties and bring greater cooperation.

The Paris Climate Agreement, signed by 178 parties, is considered the strongest climate agreement of the century. It addresses global climate change through effective mechanisms. It strengthens cooperation and also requires countries, regions, and cities to act in concert and work together [12]. The Paris Agreement states that its long-term goal is to 'substantially reduce global greenhouse gas emissions to limit the global temperature increase in this century to 2 degrees Celsius while pursuing efforts to limit the increase even further to 1.5 degrees' [13]. The Paris Agreement was officially in force on 4 November 2016.

\subsubsection{1 advantages}

Multilateral mechanisms can provide a platform for contacting more participants, and encouraging data and experience sharing from multiple channels. One of the best examples of multilateral mechanisms is the UN Climate Change Conference Paris 2015 (the Convention). There are 196 countries and regions that have signed agreements on temperature control, and 150 countries that have submitted emission reduction plans. The Convention stipulates that all countries should work together, with developed countries taking the lead in development and providing support and assistance to developing countries [13]. And the ultimate point of the Convention is to promote the maximum use of resources.

Developing countries will receive more help in the multilateral mechanism. At the UN Climate Conference Paris 2015, it was clearly stated that there would be $\$ 100$ billion in financing to support greenhouse improvement in the underdeveloped regions by 2020 . At the same time, there will be technology transfer and other measures to help some developing countries to achieve carbon neutrality. Some developing countries can hardly cope with the greenhouse effect by their strength, therefore, this kind of assistance is very important for them.

\subsubsection{2 disadvantages}

Different standards of measurement can make it difficult for countries to agree, which can undermine cooperation. Under the Paris Agreement, nations are expected to do their best to meet their targets [13], but it is difficult to define 'best' standards for different countries. The best efforts that some countries believe they can achieve may not resonate with other countries. Disputes may arise from this.

Multilateral mechanism cooperation may lack a strong regulatory mechanism. The Paris Agreement is an example of a mature multilateral mechanism that has relevant legal treaties and effects in order to maintain the effectiveness of the results. There are also mechanisms in place to facilitate compliance and implementation of the agreement. However, the expression in the relevant terms in the Agreement is only to facilitate, not to monitor. This makes the role of the Agreement lack relevant strength. In addition, the mechanism committee established is composed of experts whose roles are only non-confrontational and non-punitive [13]. This has given some countries the so-called freedom. For example, 2019 saw the US formally withdrawing from the Paris Agreement, which has had a huge impact on the global environmental governance.

\subsubsection{Complementarity of bilateral and multilateral mechanisms}

The ideal state of affairs for a country's institutional model of external engagement is to leverage the strengths of both types of mechanisms and avoid their respective weaknesses. Green Belt and Road Initiative shows a good example on how these two mechanisms can be used in combination.

Green Belt and Road Initiative is a cooperation based on the concept of environmental protection proposed by China based on the construction of the Belt and Road Initiative (BRI). In particular, it provides support and assistance for the green development of countries along the Belt and Road Initiative.

With regards to the multilateral mechanisms, the Green Belt and Road Initiative has helped the BRI countries to participate in clean energy development and to achieve green development. Dubai's 700MW solar power plant project is a tripartite investment between Dubai, China, and Saudi Arabia. This project can help Dubai to reduce 1.4 million tons of carbon emissions per year [14]. Meanwhile, one can find many bilateral mechanisms in the Green Belt and Road Initiative. For example, in 2015 the Chinese President and the Pakistani Prime Minister signed the Memorandum of Understanding and Cooperation on the Joint Development of Hydropower Projects in Pakistan to assist in clean energy development in Pakistan [15].

Sometimes, bilateral mechanisms are developed from the multilateral mechanism. The United Nations Framework Convention on Climate Change (UNFCCC) and many bilateral mechanisms around it shows an example. UNFCCC is a convention adopted by the United Nations in 1992 and entered into force in 1994. It calls on countries to limit their greenhouse gas emissions based on the principle of 'common but differentiated 
responsibilities'. Developed countries are required to reduce greenhouse gas emissions and provide assistance to developing countries. Developing countries have a lesser responsibility and do not have legally binding control obligations.

China actively signed the UNFCC and is committed to working with various countries to improve the environment and climate. On this basis China also signed the U.S.-China Joint Statement on Climate Change with the United States.

Sometimes, bilateral mechanisms serve as foundations for the multilateral mechanism. For example, in order to ensure the successful signing of the Paris Agreement, in 2015 China, India, Brazil, the European Union and France issued the China-India Joint Declaration on Climate Change, the China-Brazil Joint Declaration on Climate Change, the China-EU Joint Declaration on Climate Change and the China-France Head of State Joint Declaration on Climate Change, which laid the foundation for the successful signing of the Paris Agreement.

\section{CONCLUSION}

Few previous articles have linked bilateral and multilateral mechanisms to environmental issues, the role of which is essential for the global environment governance. This research fills this gap by comparing bilateral and multilateral mechanisms through China's environmental policies. As the research has demonstrated, bilateral and multilateral mechanisms have given China more ideas and tools to deal with environmental issues and formulate environmental policies. The paper also discusses and analyzes in detail the reasons for China's shift from bilateral mechanisms to a coexistence of bilateral and multilateral mechanisms. Both bilateral mechanisms and multilateral mechanisms have advantages and disadvantages. It would be very beneficial to leverage the advantages of both mechanisms simultaneously. In this paper, this possibility has been discussed and confirmed.

For studying bilateral and multilateral mechanisms, China is a good case study. At the beginning of the twenty-first century, China preferred policies under bilateral mechanisms, while around 2010, China's policies began to shift towards the co-existence of bilateral and multilateral mechanisms. China, the United States, and the European Union have always had a significant influence on the international community.

However, this study is limited as it only chooses to study China's environmental policy as a single case study, which may not be widely applied to other countries. Different countries will have various environmental policies according to their development interests. Developed countries usually have more diversified environmental policies than developing countries. And according to their location and their ecological environment, each country will have different interests. Different policies and mechanisms will be used for various demands. For the sake of comprehensiveness, more cases should be selected for analysis.

It can be expected that the simultaneous application of both mechanisms will benefit international relations in the field of environmental issues if those mechanisms are properly designed. The question is how to make the best of both mechanisms. Optimistically, countries will learn their own lessons through development and explorations.

\section{REFERENCES}

[1] Keohane, R. O. (1990). Multilateralism: An Agenda for Research. International Journal, 45(4), 731-764. https://doi.org/10.2307/40202705

[2] Ruggie, J. G. (1992). Multilateralism: the Anatomy of an Institution. International Organization, 46(3), 561-598. http://www.jstor.org/stable/2706989

[3] Huang Yongguang. (2001). Multilateral Mechanisms and the East Asian Security Order. Pacific Journal (03),90-96. doi:10.14015/j.cnki.10048049.2001.03.010

[4] Asuka, J. (2012). Future Climate Regime and Climate Unilateralism. Institute for Global Environmental Strategies. http://www.jstor.org/stable/resrep00900

[5] Zhang Rui, Zhou Yuanbing \& Xiang Junyong. (2019). Reanalysis of Bilateral and Multilateral Mechanisms of International Energy. Journal of North China Electric Power University (JCR-SSCI) (04), 1-9. doi:10.14092/j.cnki.cn113956/c.2019.04.001

[6] Gao Xiang, Wang wentao \& Dai Yande. (2012). Impact of Multilateral Mechanisms Outside the UNFCCC on the UNFCC. World Economy and Politics(04),59-71+157-158. doi:CNKI:SUN:SJJZ.0.2012-04-006.

[7] Hu Wei. (2010). Cooperation and Conflict: China and the United States in Global Environmental Governance (Master's thesis, Zhejiang University). https://kns.cnki.net/KCMS/detail/detail.aspx?dbna $\mathrm{me}=\mathrm{CMFD} 2010 \&$ filename $=2010099451 . \mathrm{nh}$

[8] China State Council. (2014). U.S.-China Joint Statement on Climate Change. Beijing: NDRC CHINA.

[9] China-Europe Environment Cooperation Project. (2021). [Ebook]. Retrieved from https://www.documents.clientearth.org/wpcontent/uploads/library/2021-06-15xxxxxxxxxxxxxxxxxxxxxxxxxxxxxxxxxxxx-cecn.pdf 
[10] China State Council. (2014). U.S.-China Joint Statement on Climate Change. Beijing: NDRC CHINA.

[11] United Nations. (1992). United Nations Framework Convention on Climate Change. Rio de Janeiro.

[12] Zhang, Z. (2013). Energy and Environmental Issues and Policy in China. Fondazione Eni Enrico Mattei (FEEM). http://www.jstor.org/stable/resrep00994

[13] United Nations. (2015). The Paris Agreement. Paris.

[14] China-Europe Environment Cooperation Project. (2021). [Ebook]. Retrieved from https://www.documents.clientearth.org/wpcontent/uploads/library/2021-06-15xxxxxxxxxxxxxxxxxxxxxxxxxxxxxxxxxxxx-cecn.pdf

[15] Silk Road Fund Partners with Three Gorges Group to Invest in Hydropower Projects in Pakistan. (2021). Retrieved 19 October 2021, from http://www.silkroadfund.com.cn/cnweb/19930/199 38/20122/index.html 